\title{
Determination of Film Thickness Through Simulation of Vickers Hardness Testing
}

\author{
Maxwell Santana Libório ${ }^{a}$, Avelino Manuel da Silva Dias ${ }^{a, *}$, Roberto Martins Souza ${ }^{b}$ \\ ${ }^{a}$ Department of Mechanical Engineering, Federal University of Rio Grande do Norte (UFRN), 59072- \\ 970, Natal, RN, Brazil \\ ${ }^{b}$ Department of Mechanical Engineering, Polytechnic School of University of São Paulo (USP-SP), \\ 05508-010, São Paulo, SP, Brazil
}

Received: December 23, 2015; Revised: September 28, 2016; Accepted: March 12, 2017

\begin{abstract}
In recent decades, changes in the surface properties of materials have been used to improve their tribology characteristics. However, this improvement depends on the process, treatment time and, essentially, the thickness of this surface film layer. Physical vapor deposition $(P V D)$ has been used to increase the surface hardness of metallic materials. The aim of the present study was to propose a numerical-experimental method to assess the thickness $(l)$ of films deposited by PVD. To reach this objective, Vickers experimental hardness data $\left(H_{V}\right)$ assays were combined with numerical simulation to study the behavior of this property as a function of maximum penetration depth of the indenter $\left(h_{\max }\right)$ into the film/substrate conjugate. A strategy was developed to combine the numerical results of the $H$ $\mathrm{x} h_{\max } / l$ curve with Vickers experimental hardness data $\left(H_{V}\right)$. This methodology was applied to a TiNcoated M2 tool steel conjugate. The mechanical properties of the studied materials were also determined. The thickness results calculated for this conjugate were compatible with their experimental data.
\end{abstract}

\section{Keywords: Thin Films, Finite Element, Vickers Hardness, Thickness measurement}

\section{Introduction}

Thin films are currently been used in a significant number of industrial applications and research, e.g., to increase surface resistance to corrosion and improve the tribological properties of mechanical components, to increase the surface strength of cutting tools, and to introduce a biocompatible layer in medical implants and electromagnetic devices. ${ }^{1,2}$

To this end, knowledge of the mechanical properties of conjugates composed of thin films deposited onto metallic substrates is necessary to predict the mechanical behavior of these coated systems and their numerous applications. ${ }^{2}$ However, in such conjugates, traditional standard tensile testing cannot be applied to obtain their mechanical properties. The instrumented indentation test has been increasingly applied to determine the mechanical properties of these film/substrate conjugates. ${ }^{3-9}$ This technique can be used to characterize the mechanical properties of thin films, as it characterizes the material locally from only a small volume. Furthermore, sample preparation to apply indentation tests is usually simple. Two different procedures can be applied to characterize these conjugates. The first consists of conducting several instrumented indentation tests. ${ }^{3-4,6}$ The second involves performing a few indentation tests, followed by a numerical methodology to determine these mechanical properties. $^{7-8}$ The mechanical properties of the materials used in the present study were obtained by applying the latter methodology, developed by Dias and Godoy (2010). ${ }^{7}$ I.e., these properties were determined in the present work by combining experimental and numerical Berkovich testing.

*e-mail: avelino.dias@ct.ufrn.br
Among the numerous mechanical properties of these conjugates, surface hardness is one of the most important, given that it reveals the resistance of the material to penetration, and has recently been linked to the useful life of the film, primarily in relation to its delamination. ${ }^{10-11}$ However, conjugates composed of thin films have limitations in the conventional use of surface hardness. Whether due to the geometry of the indenter or to the capacity of the film to fracture during experimental testing, the surface hardness of these conjugates depends on the thickness of the film and on the load used during the hardness test. ${ }^{5,12}$

The instrumented indentation technique has also been used to determine the hardness of these conjugates composed of thin films deposited on metallic substrates. ${ }^{5}$ Depending upon the conjugate film with substrate, the effects of their mechanical properties tend to establish a critical ratio between maximum penetration depth $\left(h_{\max }\right)$ and film thickness $(l)$, so that the substrate does not interfere in the assessment of film properties. ${ }^{13-14}$ On the other hand, determining film thickness becomes difficult due to the need for specific instruments and costly, protracted tests, such as scanning electron microscopy (SEM) and Calowear testing, in addition to instrumented indentation testing. ${ }^{15-16}$

Accordingly, the present study used the Finite Element Method (FEM) to simulate the indentation testing in a conjugate composed of Titanium Nitride (TiN) film deposited by plasma processing onto a tool Steel (M2). In an initial step, by simulating the Berkovich indentation testing and using the methodology proposed by Dias and Godoy (2010), the mechanical properties of this conjugate were determined. ${ }^{7}$ Then, in a second step, the simulation of Vickers indentation was conducted to numerically assess the penetration depth 
as a function of the hardness. Finally, it was proposed a simple experimental-numerical methodology to determine the thickness of these films by comparing this numerical result with experimental Vickers hardness testing.

\section{Methodology}

To achieve the objective of the present study, a numerical analysis was developed, followed by an experimental hardness test in the film/substrate conjugate (TiN/M2).

\subsection{Numerical procedure}

Numerical analysis was developed with MARC ${ }^{\mathrm{TM}}$ Finite Elements commercial software (2015). ${ }^{17}$ In this study, discrete models were used to reproduce the contact between the Vickers and Berkovich indenter and the sample. In accordance with Dias and Godoy (2010), these models did not consider the problems of indentation pile-up or sink-in. ${ }^{7}$ According to the literature, among others parameters, these features depend on the coefficient of friction between the indenter and the sample, which in the present study was considered to be zero, as friction has little influence on indentation load. ${ }^{18-19}$

Both the Vickers and Berkovich indenters were modeled as a rigid plate, with a square or triangular base, respectively., ${ }^{7,20}$ The sample materials were considered isotropic and homogeneous with elastic-plastic behavior, as per Equation (1). This is a classical expression described in the literature, for example by Hosford and Caddell (1993). ${ }^{21}$ The samples were modeled with threedimensional elements, as used by Libório (2015). ${ }^{12}$ As boundary conditions, the nodal displacements at the base of the sample were restricted. The other conditions were established using 1/4 of the symmetry for the Vickers model and 1/6 for the Berkovich model. ${ }^{7,20,22}$ The Vickers analysis was modelled with a mesh with 17,480 nodes and 15,930 brick elements. The Berkovich model used a mesh with 6,584 nodes and 5,472 brick elements.

$$
\sigma=\left\{\begin{array}{l}
E \varepsilon^{1.0}(\text { elastic phase }) \\
\left.K \varepsilon^{n} \text { (plastic phase }\right)
\end{array}\right.
$$

In Equation (1), $\sigma, \varepsilon$ and $E$ are effective stress, effective strain and Young's Modulus, respectively. $K$ and $n$ are constants that describe the strain-hardening characteristics of the material, $n$ being its coefficient. ${ }^{8,11,12,18,20,23}$ Table 1 shows the Young's Modulus $(E)$ and the Poisson coefficient (v) that were obtained in the literature and were used in the present simulation. ${ }^{24-25}$ The values of $K$ and $n$ were obtained from Equation (2), by combining the experimental results of the Berkovich test with numerical simulations. ${ }^{7}$

$$
\begin{aligned}
& \sigma=\sigma_{0}\left(\frac{\varepsilon}{\varepsilon_{0}}\right)^{n} \\
& \text { where, } K=\frac{\sigma_{0}}{\varepsilon_{0}^{n}} \\
& \text { and, } n=\frac{\ln \left(\frac{\sigma_{u}}{\sigma_{0}}\right)}{\ln \left(\frac{\varepsilon_{u}}{\varepsilon_{0}}\right)}
\end{aligned}
$$

Table 1. Mechanical properties of M2 tool steel substrate and TiN film. ${ }^{24-25}$

\begin{tabular}{lcc}
\hline Material & $E(G P a)$ & $v$ \\
\hline M2 & 200 & 0.30 \\
TiN & 120 & 0.25
\end{tabular}

In this Equation (2), $\varepsilon_{u}$ is the ultimate strain, $\varepsilon_{o}$ is the yield strain, $\sigma_{u}$ is the ultimate strength, $\sigma_{o}$ is the yield stress and $n$ is the strain-hardening coefficient, as seen previously. These values were obtained using the experimental numerical methodology developed by Dias and Godoy (2010). ${ }^{7}$

\subsection{Determination of film thickness}

After determining the mechanical properties of both the film (TiN) and substrate (M2), Vickers hardness testing was simulated on the surface of the TiN/M2 conjugate. Given that the thickness of the Titanium Nitride film was initially unknown, in the simulations of the Vickers test a fixed value for penetration depth was assigned as 0.8 $\mu \mathrm{m} .{ }^{12}$ The aim was to obtain a surface hardness range that reflects the contribution from the film and the substrate. This Vickers hardness was computed as numerical load $(P)$ divided per area of the residual impression on surface $\left(A_{r}\right)$ using the known Equation (3). ${ }^{20,26} \mathrm{Next}$, the film thickness $(l)$ was varied in the numerical analysis and the hardness behavior was determined as a function of the ratio between maximum penetration depth and film thickness $\left(h_{\max } / l\right)$. The values adopted for the ratio in the different simulations of the Vickers test are presented in Table 2. Figure 1 illustrated one of the meshes used in the present work. In this case, the indentation depth was $2.5 \mu \mathrm{m}$.

$$
H_{V}=P / A r
$$

Originpro $^{\mathrm{TM}} 8$ software was used to analyze the numerical behavior of the relationship between numerical Vickers hardness $\left(H_{V}\right)$ and the $h_{\text {max }} / l$ ratio. ${ }^{27}$ The power curve obtained by this analysis was exponential as shown in Equation (4). Constants $A_{1}, A_{2}, t_{1}, t_{2}$ and $H_{V 0}$ are the coefficients of this expression. The ordinate axis represents the numerical Vickers hardness values and the $x$ axis (abscissas) the independent variable $\left(h_{\max } / l\right)$. In this expression, constant $H_{V 0}$ was included in order to fit the curve, without further considerations regarding its physical meaning, at least at this point.

$$
H_{V}=A_{1} e^{-\frac{x}{t_{1}}}+A_{2} e^{-\frac{x}{l_{2}}}+H_{V 0}
$$

This expression was one of the main results obtained in this study, due to the fact that this numerical $H_{V}$ curve as a function of $h_{\max } / l$ was used to determine film thickness by entering the experimental value of Vickers hardness. The flow chart in Figure 2 revealed how to combine numerical and 
Table 2. Used values in a FE simulation for different thickness $(l)$ and for the ratio $h_{\max } /$.

\begin{tabular}{lccccccc}
\hline$l(\mathrm{~nm})$ & 500 & 600 & 700 & 900 & 1,000 & 1,200 & 1,350 \\
$h_{\max } l$ & 1.6 & 1.33 & 1.14 & 0.88 & 0.8 & 0.66 & 0.59 \\
$l(\mathrm{~nm})$ & 1,600 & 1,800 & 2,000 & 2,350 & 2,500 & 3,000 & 8,000 \\
$h_{\max } l$ & 0.5 & 0.44 & 0.4 & 0.34 & 0.32 & 0.26 & 0.1 \\
\hline
\end{tabular}

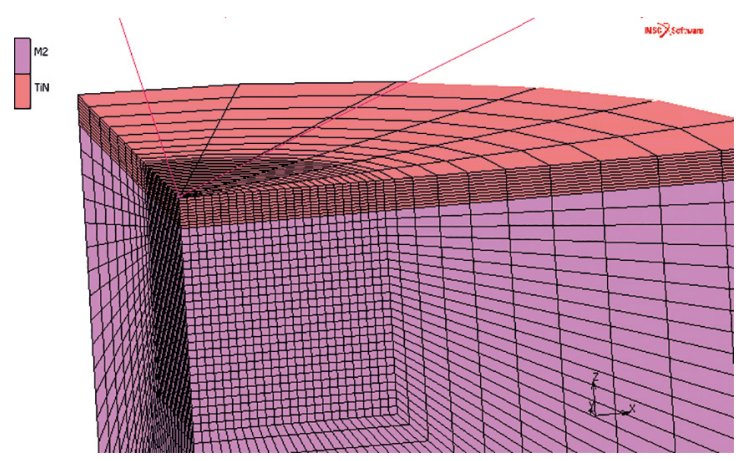

Figure 1. Numerical mesh used to simulate the Vickers indentation testing with $h_{\max } l=0.34$, using 1,308 brick elements for TiN film and 5,742 elements for M2 substrate.

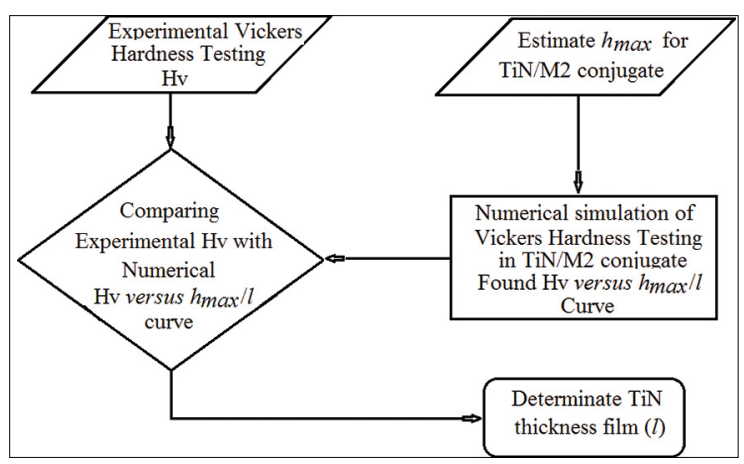

Figure 2. The flow chart used to combine numerical and experimental procedures to determinate the thickness of film.

experimental procedures to determinate the film thickness. First it was estimated the depth of penetration $\left(h_{\max }\right)$ to perform numerical simulation, varying the film thickness $(l)$ and calculating their surface hardness $\left(H_{V}\right)$. Next, it was determined the $H_{V}$ versus $h_{\max } / l$ curve. An experimental Vickers hardness was entered to the numerical curve and was obtained the film thickness.

\section{Results and Discussions}

Based on the experimental results obtained in the instrumented indentation test with a Berkovich indenter, a number of numerical simulations was carried out in order to determine the mechanical properties for of both Titanium Nitride (TiN) and M2 tool steel substrate. As discussed previously, the methodology developed by Dias and Godoy (2010) was used to find the yield limit $\left(\sigma_{o}\right)$, strain hardening exponent $(n)$ and $K$ constant for the TiN/M2 conjugate. ${ }^{7}$ Table 3 shows the mechanical properties found for the aforementioned materials. Given the doubts still existing in the literature when an experimental numerical methodology is used to determine the mechanical properties of surface films, it was decided to verify and validate these mechanical properties. For example, an analysis of the result obtained for the yield limit of M2 steel indicated that it was within the range of values indicated in the literature, that is, between $1,650 \mathrm{MPa}$ and 3,500 MPa. ${ }^{25}$ Furthermore, Table 4 shows a comparison between experimental and numerical values for the indentation load and penetration depth of the Berkovich test in the M2 tool steel substrate. The difference between the mean maximum experimental load obtained with a mean penetration depth of $2,860 \mathrm{~nm}$ was about $1 \%$ when compared with the respective numerical value $\left(P_{m u m}\right)$, for a penetration depth of $2,900 \mathrm{~nm}$. In summary, this numerical result indicates that the simulation of the Berkovich test is compatible with its experimental behavior for the substrate.

Obviously, the comparison between the experimental and numerical values of Berkovich indentation testing was not used for TiN film, given that film thickness was unknown. According to the literature, film thickness influences the mechanical response of the conjugate when submitted to the indentation test. ${ }^{4,23,28}$ In other words, the simulations performed assessed not only film behavior, but also that of the M2/TiN conjugate.

The mechanical proprieties in Table 3, as well as the properties exhibited in Table 1, served as input parameters for the FE software. ${ }^{17}$ After determination of these properties of the conjugate, fourteen simulations were conducted to represent the Vickers hardness test for the M2/TiN conjugate. In these simulations, the $h_{\max } / l$ ratios in Table 2 were used. Figure 3 shows the results of these simulations in terms of Vickers hardness behavior as a function of the $h_{\max } / l$ ratio. Qualitative assessment of the values shown in this figure demonstrates that for low $h_{\text {max }} / l$ ratio values the superficial hardness exhibits low contribution from the substrate,

Table 3. Input mechanical properties used to simulate the conjugate TiN/M2.

\begin{tabular}{lccc}
\hline Material & $K(M P a)$ & $n$ & $\sigma_{0}(M P a)$ \\
\hline M2 & $4,995.13$ & 0.12 & 2,874 \\
TiN & $28,115.66$ & 0.42 & $6,441.0$ \\
\hline
\end{tabular}

Table 4. Numerical and experimental measures of $P$ values in relation to $h_{\max }$ and the relationship between the experimental and numerical load value for M2 steel.

\begin{tabular}{lllll}
\hline$h_{\text {num }}(\mathrm{nm})$ & $\bar{h}_{\max }(\mathrm{nm})$ & $\bar{P}_{\text {exp }}(\mathrm{mN})$ & $P_{\text {num }}(\mathrm{mN})$ & Pdifference \\
\hline 2,900 & 2,857 & 1,257 & 1,270 & $1.0 \%$ \\
\hline
\end{tabular}




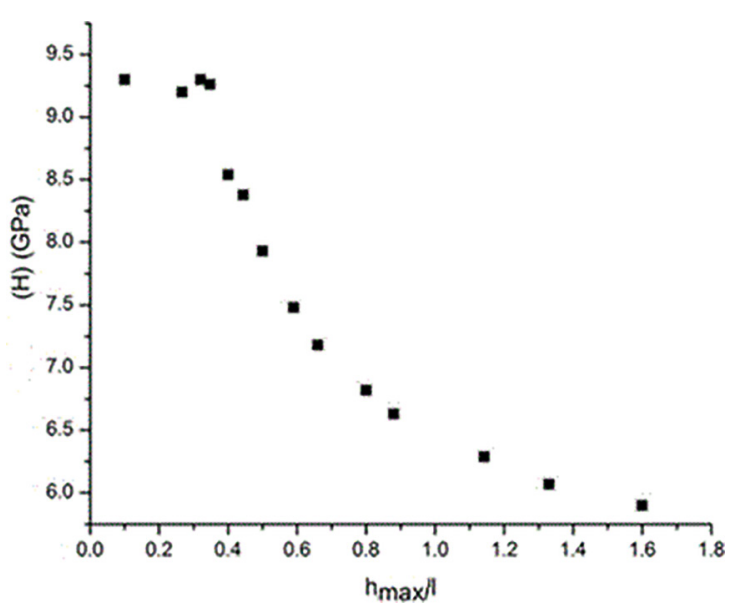

Figure 3. Numerical results for Vickers hardness in function of $h_{\max } / l$ ratio for the conjugate M2/TiN.

remaining approximately constant. ${ }^{4,23}$ Braz (2012) determined the experimental Vickers Hardness at about 11.0 GPa for the same TiN film. This author deposited the TiN film in M2 steel substrate by plasma process using the Plasma Laboratory at the Federal University of Rio Grande do Norte. ${ }^{29}$ Thus, the numerical superficial hardness exhibited a value close to that of the TiN film. On the other hand, the hardness value for large $h_{\max } / l$ ratios converges to that of the M2 steel substrate. Once again, in the literature, the $H_{V}$ of M2 steel varies between 2.0 and $7.0 \mathrm{GPa}^{25}$

In 2006, Gao et al., using the expanding cavity model for strain hardening material that follows Hollomon's power law relationship, developed different relationships between hardness $\left(H_{V}\right)$, yield strength $\left(\sigma_{o}\right)$, strain-hardening exponent $(n)$, and Young's modulus $(E)$ for Vickers indentation. ${ }^{30}$ The Vickers hardness calculated from the Gao et al (2006) formulations was then compared to the values obtained in the present work. From the expression for elastic power-law hardening materials (Equation 5) and using the mechanical properties (Tables 1 and 3 ) for the TiN film, the estimated Vickers hardness was $12.9 \mathrm{GPa}$. For the M2 steel substrate, the Vickers Hardness was 7.4 GPa, using the expression for elastic-perfectly plastic materials (Equation 6). In these two expressions, $\alpha$ is the half-included angle of the equivalent cone to the Vickers indenter. ${ }^{2,30}$ The TiN hardness, calculated from Equation 5, differs 27\% from the results exhibited in Figure 3. This difference could be explained by the fact that no hypothesis in terms of indentation morphology (neither pile-up, nor sink-in) was considered in this work or because Gao et al (2006) formulations are better when they were applied in bulk materials, or both. ${ }^{30}$ The M2 hardness obtained, from Equation 6, differs 15\% from the one determined here. This good result for the M2 steel also confirmed that this material has a small elastic recovery.

$$
\begin{gathered}
H_{V}=\frac{2}{3}\left\{\left(1-\frac{1}{n}\right)+\left(\frac{3}{4}+\frac{1}{n}\right)\left(\frac{1}{3} \frac{E}{\sigma_{0}} \cot \alpha\right)^{n}\right\} \sigma_{0} \\
H_{V}=\frac{2}{3}\left\{\frac{7}{4}+\ln \left(\frac{1}{3} \frac{E}{\sigma_{0}} \cot \alpha\right)\right\} \sigma_{0}(6)
\end{gathered}
$$

Data from Figure 3 were analyzed using the Originpro 8 program $^{27}$, disregarding the first three points, since they may be influenced by film hardness, resulting in the graph depicted in Figure 4. This figure also illustrates the experimental hardness values reported by Almeida (2008) for the same sample of the M2/TiN conjugate. ${ }^{31}$ The first mean hardness value was $8.9 \mathrm{GPa}$ for a load of $0.49 \mathrm{~N}$ (50 gf). The second was $8.25 \mathrm{GPa}$ for a load $0.98 \mathrm{~N}$ (100 gf).

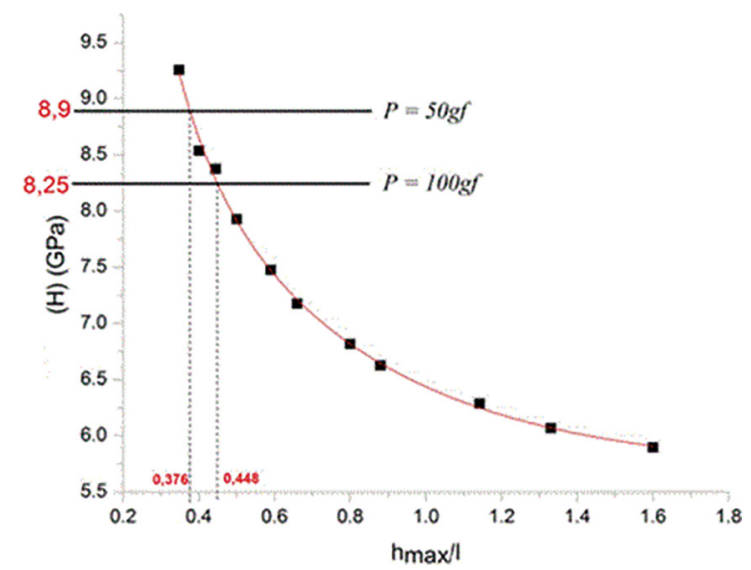

Figure 4. Numerical curve for Vickers hardness in function of $h_{\max } / l$ ratio for the studied conjugate.

Applying the algorithm shown in Figure 2, for a load of $0.49 \mathrm{~N}$ and Vickers hardness of $8.90 \mathrm{GPa}$, resulted in a thickness of 2,130 nm for TiN film. A thickness of 1,780 nm was obtained when the hardness of $8.25 \mathrm{GPa}(P=0.98 \mathrm{~N})$ was used. These values were compared with the thickness of this TiN film in this sample, also found by Almeida (2008) $)^{31}$, using the Calowear Testing. Table 5 illustrates the difference obtained by the proposed methodology in determining film thickness, compared to the experimental value obtained by Almeida (2008). The first thickness value showed a difference of around $1.4 \%$ in relation to the experimental value. The second value obtained from the higher hardness exhibited a difference of around $17.7 \%$. The better film thickness result is due to the lower load in the hardness test. In this case, the Vickers indenter had lower penetration into the TiN/M2 conjugate. It is important to remember that in the simulated test a penetration depth of $0.8 \mu \mathrm{m}$ was used, corresponding to $38 \%$ of film thickness experimentally determined by Almeida (2008). ${ }^{31}$

Table 5. Comparison between the thickness values for the TiN film obtained by proposed method with the experimental value obtained through the assay Calowear (Almeida, 2008).

\begin{tabular}{lcc}
\hline Vickers Hardness (load) & $\begin{array}{c}8.9 \mathrm{GPa} \\
(0.98 \mathrm{mN})\end{array}$ & $\begin{array}{c}8.25 \mathrm{GPa} \\
(0.49 \mathrm{mN})\end{array}$ \\
\hline Numerical thickness & $1,783 \mathrm{~nm}$ & $2,130 \mathrm{~nm}$ \\
Experimental thickness $^{31}$ & \multicolumn{2}{c}{$2,100 \mathrm{~nm}$} \\
Error & $17.7 \%$ & $1.4 \%$ \\
\hline
\end{tabular}




\section{Conclusions}

The 3D finite element (FE) model developed in the present work was able to adequately represent the general behavior of the Berkovich and Vickers indentation testing. Also, this study employed a methodology that combined experimental Vickers hardness tests with their numerical simulation in order to obtain the thickness of a titanium nitride film deposited by PVD onto an M2 tool steel.

Experimental procedures were carried out to obtain the mechanical properties of the materials under study. This procedure, developed by Dias and Godoy $(2010)^{7}$, was able to determine the mechanical properties for both the substrate (M2 steel) and the film (TiN). These mechanical properties exhibited consistent results when compared to literature data. Also, the numerical simulation of the Berkovich indentation test for a sample of M2 steel substrate reproduced the behavior of the same experimental assay. It is important to emphasize that the use of numerical analysis in combination with indentation testing confirmed promising results in assessing mechanical properties.

Knowing the mechanical properties of the film and substrate conjugate, numerical simulations were used to obtain hardness behavior as a function of the variation in film thickness or as a function of the $h_{\max /}$ ratio. Next, data processing software Originpro ${ }^{\mathrm{TM}} 8(2008)^{27}$ was applied to obtain a curve that characterizes Vickers hardness as a function of film thickness or the $h_{\max } / l$ ratio. Finally, film thickness was determined by combining the results of Vickers hardness tests and their Vickers hardness curve for the M2/TiN conjugate. Numerical results showed that this methodology produced good results for the sample under study and may represent a new direction in relation to other burdensome procedures for determining film thickness, such as SEM and Calowear testing.

We intend to improve this methodology by introducing a convergence test in the flowchart of Figure 2. Another possible optimization to be incorporated into this methodology would be to compare the experimental numerical behavior of Berkovich hardness, given that this experimental test was conducted a priori to determine the mechanical properties of the TiN/Ti system required for numerical simulation.

\section{Acknowledgments}

The authors gratefully acknowledge the funding by CNPq (Brazilian Council for Scientific and Technological Development), under grants Universal 14/2011, process 484818/2011-2, and Universal 14/2014, process 460943/2014-6. One of them also thanks for the CAPES (Higher Coordination Brazilian Agency for Scientific and Educational Training), through process BEX 6569-14.

\section{References}

1. Le Bourhis E. Indentation mechanics and its application to thin film characterization. Vacuum. 2008;82(12):1353-1359.

2. Moussa C, Bartier O, Mauvoisin G, Pilvin P, Delattre G. Characterization of homogenous and plastically graded materials with spherical indentation and inverse analysis. Journal of Materials Research. 2012;27(1):20-27.

3. Zeng K, Chiu C. An analysis of load-penetration curves from instrumented indentation. Acta Materialia. 2001;49(17):3539-3551.

4. Oliver WC, Pharr GM. Measurement of hardness and elastic modulus by instrumented indentation: Advances in understanding and refinements to methodology. Journal of Materials Research. 2004;19(1):3-20.

5. Souza GB, Foerster CE, Silva SLR, Serbena FC, Lepienski CM, Santos CA. Hardness and elastic modulus of ion-nitrided titanium obtained by nanoindentation. Surface and Coatings Technology. 2005;191(1):76-82.

6. Fischer-Cripps AC. Critical review of analysis and interpretation of nanoindentation test data. Surface and Coatings Technology. 2006;200(14-15):4153-4165.

7. Dias AMS, Godoy GCD. Determination of Stress-Strain Curve through Berkovich Indentation Testing. Materials Science Forum. 2010;636-637:1186-1193.

8. Pulécio SAR, Farias MCM, Souza RM. Finite element and dimensional analysis algorithm for the prediction of mechanical properties of bulk materials and thin films. Surface and Coatings Technology. 2010;205(5):1386-1392.

9. Rodríguez SA, Alcalá J, Souza, RM. The reduced modulus in the analysis of sharp instrumented indentation tests. Journal of Materials Research. 2012;27(16):2148-2160.

10. Sun Y, Bloyce A, Bell T. Finite element analysis of plastic deformation of various TiN coating/substrate systems under normal contact with a rigid sphere. Thin Solid Films. 1995;271(12):122-131.

11. Araújo R, Dias AMS. Numerical Evaluation of Strength in the Interface during Indentation Spherical Testing in Thin Films. Materials Sciences and Applications. 2014;5(3):149-157.

12. Libório MS. Metodologia numérico-experimental para avaliação da espessura de filmes de nitreto de titânio obtidos por processamento a plasma. [Dissertation]. Natal: Federal University of Rio Grande do Norte; 2015. 73 f. [in Portuguese]

13. Bressan JD, Tramontin A, Rosa C. Modeling of nanoindentation of bulk and thin film by finite element method. Wear. 2005;258(14):115-122.

14. Pelletier H, Krier J, Mille P. Characterization of mechanical properties of thin films using nanoindentation test. Mechanics of Materials. 2006;38(12):1182-1198.

15. Le Bourhis E, Patriarche G. Plastic deformation of III-V semiconductors under concentrated load. Progress in Crystal Growth and Characterization of Materials. 2003;47(1):1-43.

16. Nunes Filho A. Influência das espécies ativas na absorção de intersticiais durante a carbonitretação a plasma do Ti. [Dissertation]. Natal: Federal University of Rio Grande do Norte; 2011. 95 f. [in Portuguese] 
17. Marc ${ }^{\mathrm{TM}}$ MSC. Software Corporation. Volume A: Theory and User Information; User's Manual. Available from: $<\mathrm{http}$ ://www.mscsoftware. com>. Access in: 31/08/2015.

18. Mata M, Alcalá J. The role of friction on sharp indentation. Journal of the Mechanics and Physics of Solids. 2004;52(1):145-165.

19. Araújo R, Dias AMS, Godoy GCD. Numerical study of the influence of friction coefficient for indentation testing in thin films. Matéria (Rio de Janeiro). 2013;18(1):55-66. [in Portuguese]

20. Dias AMS, Modenesi PJ, Godoy GCD. Computer simulation of stress distribution during Vickers hardness testing of WC-6Co. Materials Research. 2006;9(1):73-76.

21. Hosford WF, Caddell RM. Metal Forming: Mechanics and Metallurgy. New Jersey: Prentice Hall PTR; 1993.

22. Zhang W, Subhash G. An elastic-plastic-cracking model for finite element analysis of indentation cracking in brittle materials. International Journal of Solids and Structures. 2001;38(34-35):5893-5913.

23. Souza GB, MikowskiA, Lipienski CM, Foerster CE. Indentation hardness of rough surfaces produced by plasma-based ion implantation process. Surface and Coatings Technology. 2010(18-19);204:3013-3017.

24. ShanZ, Sitaraman SK. Elastic-plastic characterization of thin films using nanoindentation technique. Thin Solid Films. 2003;437(1-2):176-181.
25. Matweb. Material Property Data. Available from: <http://www. matweb.com>. Access in: 23/06/2015.

26. Souza SA. Ensaios Mecânicos de Materiais Metálicos: Fundamentos teóricos e práticos. $5^{\mathrm{a}}$ Ed. São Paulo: Edgard Blücher; 2000. [in Portuguese]

27. OriginLab ${ }^{\circ}$ Corporation. OriginPro 8 SR 4 (2008). Available from: $<$ http://www.originlab.com>. Access in: 14/02/2015.

28. Chen J, Bull SJ. Assessment of the toughness of thin coatings using nanoindentation under displacement control. Thin Solid Films. 2006;494(1-2):1-7.

29. Braz DC. Tratamento termoquímico do titânio auxiliado por plasma de Ar-N2-O2. [Dissertation]. Natal: Federal University of Rio Grande do Norte; 2011. 97 f. [in Portuguese]

30. Gao XL, Jing XN, Subhash G. Two new expanding cavity models for indentation deformations of elastic strain-hardening materials. International Journal of Solids and Structures. 2006;43(7-8):2193-2208.

31. Almeida EO. Construção, caracterização e aplicação de eletrodos para descarga por arco de cátodo oco. [Thesis]. Natal: Federal University of Rio Grande do Norte; 2008. 248 f [in Portuguese] 